\title{
Addressing the Magnetic Properties of Sub-Monolayers of Molecular Nanomagnets by X-ray Magnetic Circular Dichroism
}

\author{
F. Moro ${ }^{1 *}$, V. Corradini ${ }^{2}$, M. Evangelisti ${ }^{3}$, R. Biagi ${ }^{2}$, V. De Renzi ${ }^{2}$, U. del Pennino², \\ J. C. Cezar ${ }^{4}$, R. Inglis ${ }^{5}$, C. J. Milios, ${ }^{5,6}$ E. K. Brechin ${ }^{5}$ \\ ${ }^{1}$ School of Chemistry, University of Nottingham, University Park NG1 2RD, United Kingdom. \\ ${ }^{2}$ INFM-CNR S3 National Research Centre and Dipartimento di Fisica, Universita' di Modena e Reggio \\ Emilia, via G. Campi 213/A, 41100 Modena, Italy. \\ ${ }^{3}$ Instituto de Ciencia de Materiales de Aragón, CSIC - Universidad de Zaragoza, Departamento de \\ Física de la Materia Condensada, 50009 Zaragoza, Spain. \\ ${ }^{4}$ European Synchrotron Radiation Facility, BP 220, F - 38043 Grenoble Cedex, France. \\ ${ }^{5}$ School of Chemistry, University of Edinburgh, EH9 3JJ Edinburgh, United Kingdom. \\ ${ }^{6}$ Department of Chemistry, University of Crete, 710 03, Voutes, Herakleion, Greece.
}

\section{PACS numbers:}

\begin{abstract}
We report on a comparative study of electronic and magnetic properties of $\mathrm{Mn}_{6}$ molecular nanomagnets grafted on gold surface. Two derivatives with spin - ground state 4 and 12 have been functionalized with $3-\mathrm{tp}-\mathrm{CO}_{2}^{-}$(3 - thiophene carboxylate, tpc) ligands and characterized as thick films (TFs) as well as sub-monolayers (sMLs) by synchrotron - based techniques. X - ray absorption spectroscopy at the $\mathrm{Mn}-L_{2,3}$ edges shows the modification of the spectral lineshape in the sMLs with respect to the TFs suggesting that the local symmetry at the Mn sites changes once the molecules are deposited on gold surface. In spite of this the expected $\mathrm{Mn}^{\mathrm{III}}$ oxidation state is preserved. $\mathrm{X}$ - ray magnetic circular dichroism (XMCD) spectra show that the total magnetic moment is only given by spin part because of the quenched orbital moment. Moreover, variable temperature variable field (VTVH) XMCD spectra reveal an effective decrease of the Mn spin moment for both derivatives deposited on gold surface.
\end{abstract}

\footnotetext{
*Corresponding author: fabrizio.moro@nottingham.ac.uk
} 


\section{Introduction}

Among the several objects which can be realised in nanomagnetism, Molecular Nanomagnets (MNMs) have recently attracted much interest because of their potential application as molecular devices $^{1-8}$. They combine large spin - ground state with large and negative magnetic anisotropy giving rise to an anisotropy energy barrier leading to slow relaxation of the magnetization at low temperatures ${ }^{9}$. In principle these properties might be used to store information in an individual molecular unit, although it has been hindered so far by two main issues. Firstly, the superparamagnetic blocking temperature is still too low because, in the most fortunate cases (e.g. $\mathrm{Mn}_{12}{ }^{10}$ ), it reaches only $2-3 \mathrm{~K}^{9,11,12}$. This issue might be overcome by larger values of the spin - ground state and magnetic anisotropy, the latter being extremely difficult to achieve by controlled synthesis. Secondly, the retention of magnetic properties at the molecular level following the deposition of spatially isolated MNMs on substrates is far to be achieved ${ }^{13}$. The interaction with surfaces may modify the intrinsic properties of molecules leading to the reduction of the magnetic core and the removal of the hysteresis loop as already observed for $\mathrm{Mn}_{12}$ on gold ${ }^{14-16}$.

Recently a step forward towards the enhance of the anisotropy barrier was carried out with the synthesis of MNMs with general formula $\left[\mathrm{Mn}_{6}{ }_{6} \mathrm{O}_{2}(\mathrm{R}-\mathrm{sao})_{6}\left(\mathrm{O}_{2} \mathrm{CR}^{\prime}\right)_{2} \mathrm{~L}_{4-6}\right]$, (in short $\mathrm{Mn}_{6}$ ) where $\mathrm{R}=$ $\mathrm{H}$, Me or Et, $\mathrm{R}^{\prime}=\mathrm{H}$, Me, Ph etc., $\mathrm{L}=\mathrm{EtOH}, \mathrm{H}_{2} \mathrm{O}, \mathrm{MeOH}$ and $\mathrm{saoH}_{2}$ is salicylaldoxime. Milios et al ${ }^{17-}$ ${ }^{19}$ proved that the use of derivatised oxime ligands and bulky carboxylates deliberately increases the value of the spin - ground state of $\mathrm{Mn}_{6}$ - based systems from spin $S=4$ to $S=12$ in a stepwise fashion, and in - so - doing enhances the energy barrier for magnetization reversal $\left(U_{\text {eff }}\right)$ establishing a new record of $86.4 \mathrm{~K}$. The representative core of the $\left[\mathrm{Mn}_{6}{ }_{6} \mathrm{O}_{2}(\mathrm{sao})_{6}\left(\mathrm{O}_{2} \mathrm{CR}\right)_{2}(\mathrm{ROH})_{4}\right]$ derivative ${ }^{20,21}$ contains a non-planar $\left[\mathrm{Mn}_{6}{ }_{6}^{\mathrm{III}}\left(\mu_{3}-\mathrm{O}^{2-}\right)_{2}\left(\mu_{2}-\mathrm{OR}\right)_{2}\right]^{12+}$ unit of two off - set, stacked $\left[\mathrm{Mn}_{3}^{\mathrm{III}}\left(\mu_{3}-\mathrm{O}^{2-}\right)\right]^{7+}$ triangular subunits linked by two central oximato $\mathrm{O}-$ atoms (Figure 1, left panel). This class of compounds shows a ferromagnetic (FM) exchange interaction between the two antiferromagnetically (AF) coupled [ $\mathrm{Mn}_{3}{ }_{3}$ ] triangles leading to $S=4$ spin - ground state ${ }^{22}$. A number of important structural changes leading to different values of $S$ and $U_{\text {eff }}$ take place in the related complexes $\left[\mathrm{Mn}_{6}^{\mathrm{III}} \mathrm{O}_{2}(\mathrm{R}-\right.$ sao $\left.)_{6}\left(\mathrm{O}_{2} \mathrm{CR}^{\prime}\right)_{2}(\mathrm{EtOH})_{4}\left(\mathrm{H}_{2} \mathrm{O}\right)_{2}\right]$ with $\mathrm{R}=\mathrm{Et}, \mathrm{Me}, \mathrm{Ph}^{23}$, where the increased steric bulk and non planarity of the $\mathrm{R}-\mathrm{sao}^{2-}$ ligands cause a shortening (by $\sim 1 \AA$ ) of the phenolato oxygen - square pyramidal Mn distance and a severe twisting of the $\mathrm{Mn}-\mathrm{N}-\mathrm{O}-\mathrm{Mn}$ moieties within each $\mathrm{Mn}_{3}{ }_{3}^{\mathrm{III}}$ sub - unit (Figure 1, right panel). The structural changes force a switch in the dominant magnetic exchange interactions from AF to FM, thus stabilizing $S=12$ spin - ground state ${ }^{19,23}$. However, all these derivatives are characterized by weak exchange interaction $\left(J<1 \mathrm{~cm}^{-1}\right)$, leading to the population of low - lying excited states $(S=11,10, \ldots)$. This makes feasible their participation in the relaxation by 
quantum tunnelling of the magnetisation, which results in a reduction of the energy barrier. This problem has been successfully solved by further replacement of the carboxylates via simple metathesis type reactions that produces identical cores except for the $\mathrm{Mn}-\mathrm{N}-\mathrm{O}-\mathrm{Mn}$ torsion angles $\left(\alpha_{v}\right)$ which are modified according to the steric bulk of the ligand. The complexes with the largest torsion angles show an increase in the magnitude of the pairwise exchange parameter $(J)$ and an increase in the effective $U_{\text {eff }}$ up to $86.4 \mathrm{~K}^{19,23}$, i.e. notably higher than that of $\mathrm{Mn}_{12}-$ ac $(61 \mathrm{~K})^{2}$.

In our previous work ${ }^{24}$, we already succeeded in self - assembling of two $\mathrm{Mn}_{6}$ derivatives $\left[\mathrm{Mn}_{6}^{\mathrm{III}} \mathrm{O}_{2}(\mathrm{sao})_{6}\left(\mathrm{O}_{2} \mathrm{C}-\mathrm{tp}-3\right)_{2}(\mathrm{EtOH})_{4}\right](\mathbf{1})$ and $\left[\mathrm{Mn}_{6}{ }_{6} \mathrm{O}_{2}(\mathrm{Et}-\mathrm{sao})_{6}\left(\mathrm{O}_{2} \mathrm{C}-\mathrm{tpc}-3\right)_{2}(\mathrm{EtOH})_{4}\left(\mathrm{H}_{2} \mathrm{O}\right)_{2}\right]$ (2) on $\mathrm{Au}(111)$ surface. The spin - ground state of these compounds is $\mathrm{S}=4$ and $\mathrm{S}=12$, respectively. For both compounds, the functionalization is made by two $3-\mathrm{tp}-\mathrm{CO}_{2}^{-}$units pointing in opposite direction and being perpendicular to the molecular plane with the sulphur atoms in outermost position on the thiophene ring (Figure 1, top panel). This favours the direct grafting on gold surface by covalent bond with the molecular axis which should be preferentially normal to the surface. The investigation carried out by means of scanning tunnelling microscopy (STM), shown in Figure 1, and X-ray photoemission spectroscopy (XPS), suggests that $\mathrm{Mn}_{6}$ molecules preserve their integrity when deposited by liquid phase. The electronic structure of $\mathrm{Mn}_{6}$ on gold surface was also studied by means of resonant photoemission spectroscopy (ResPES) at the $\mathrm{Mn} L_{3}$ edge aimed to single out the $\mathrm{Mn}-3 \mathrm{~d}$ derived states in the valence band ${ }^{25}$. Moreover, magnetic measurements on powder pellets clearly showed that the $3-\mathrm{tp}-\mathrm{CO}_{2}{ }^{-}$functionalisation does not significantly alter the magnetic properties of both compounds ${ }^{24}$.

The magnetic characterization of grafted MNMs is the key point which needs to be addressed. Unfortunately standard magnetometry techniques do not have enough sensitivity for detecting the magnetism of sub - monolayer quantities of grafted clusters. In terms of high resolution and element selectivity, X-ray absorption spectroscopy (XAS) and magnetic circular dichroism (XMCD) are excellent tools. The analysis of isotropic XAS spectra at the $L_{2,3}$ edges of transition metals provides unambiguous information about the oxidation state and local symmetries of the selected chemical element. On the other hand, XMCD directly probes the local spin and orbital magnetic moments as well as the sign of the exchange interactions. In particular cases, the application of the XMCD sum rules allows the quantification of the orbital and magnetic moments in an element - specific way. Herein we address the structural, electronic and magnetic properties of the sub - monolayers (sMLs) and thick films (TFs) of 1 and $\mathbf{2}$ by means of XAS and XMCD measurements in order to elucidate how the molecular properties are affected after the deposition on surface. 


\section{Experiment}

The TFs of the $\mathrm{Mn}_{6}$ - tpc derivatives 1 and 2, and those of the pristine $\mathrm{Mn}_{6}$ - benz compounds, $\left[\mathrm{Mn}_{6} \mathrm{O}_{2}\left(\mathrm{O}_{2} \mathrm{CPh}\right)_{2}(\mathrm{sao})_{6}(\mathrm{EtOH})_{4}\right]$ and $\left[\mathrm{Mn}_{6} \mathrm{O}_{2}\left(\mathrm{O}_{2} \mathrm{CPh}\right)_{2}(\mathrm{Et}-\mathrm{sao})_{6}(\mathrm{EtOH})_{6}\right]$, were obtained by drop-cast procedure on a highly ordered pyrolytic graphite (HOPG) substrate. The sML samples were prepared by immersion of flame annealed gold on mica surface in a $1 \mathrm{mM}$ solution of the derivatives $\mathbf{1}$ and $\mathbf{2}$ in $\mathrm{CH}_{2} \mathrm{Cl}_{2}$ for $10 \mathrm{~min}$. After the adsorption process the samples were rinsed with proper solvent in order to remove the excess of molecular layers and quickly introduced into the sample chamber ${ }^{26,27}$. Solvent used, deposition time and solution concentration were optimized in order to get homogeneous sML distribution of isolated molecules. STM was used to check that the desired two - dimensional distribution of nanometric entities was actually achieved (Figure 1) ${ }^{24}$.

XAS measurements at the Mn $L_{2,3}$ edges were carried out at the ID08 Dragon beamline of the European Synchrotron Radiation Facility (ESRF, Grenoble, France) in Total Electron Yield (TEY) detection mode and normalized to the incident photon flux. The photon source was an Apple II undulator delivering a high flux $\left(10^{13}\right.$ photons/s) of $~ 100 \%$ of circularly polarized light.

The sML samples were fixed to a molybdenum sample holder by means of tantalum wires to ensure a proper thermal and electrical contact, and transferred into a liquid - helium cryomagnet kept under UHV ( $\left.10^{-10} \mathrm{mbar}\right)$. The direction of both impinging beam and magnetic field generated by superconducting coils are perpendicular to the sample surface ( $\mathrm{z}$ - axis). The XMCD spectrum is the difference between XAS spectra taken with the helicity of the incident photon $(\mathbf{P})$ antiparallel $\left(\sigma^{\uparrow}\right)$ and parallel $\left(\sigma^{\uparrow \uparrow}\right)$ to the field direction and normalized to the isotropic edge height. To avoid systematic

errors, $\sigma^{\uparrow \downarrow}\left(\sigma^{\uparrow \uparrow}\right)$ absorption spectrum was obtained by averaging a set of spectra recorded with the field orientated parallel and anti - parallel to the photons direction ${ }^{28}$. The beam flux was attenuated by more than 1 order of magnitude in order to avoid sample damaging induced by radiation exposure. In these conditions the spectra never showed any sign of degradation during the experiment.

\section{Results and Discussion}

XAS spectra taken with right $\left(\sigma^{\uparrow \uparrow}\right)$ and left $\left(\sigma^{\uparrow \downarrow}\right)$ circularly polarized light and the relative XMCD signal $\left(\sigma^{\uparrow}-\sigma^{\uparrow \uparrow}\right)$ at the Mn $-L_{2,3}$ edges for the TF of $\mathbf{1}$ and $\mathbf{2}$ are shown in Figure 2 and compared with the analogous $\mathrm{Mn}_{6}$ - benz derivatives (the pristine not functionalized compounds) with spin 4 and 12 (see experimental section). The 2p core hole spin - orbit interaction splits the spectrum in two parts, corresponding to $L_{3}\left(2 \mathrm{p}_{3 / 2}\right)$ and $L_{2}\left(2 \mathrm{p}_{1 / 2}\right)$ levels with a separation of about $11 \mathrm{eV}$. The $L_{2}$ edge features are more broadened than the $L_{3}$ edge, owing to the increased probability of the Coster - 
Kronig Auger decay channel. Hereafter we focus only on $L_{3}$ fine structure. The $L_{3}$ level is further split into the $t_{2 g}$ and $e_{g}$ sub - bands by the octahedral ligand field. The fine structure of the absorption edges is due to a combination of the electron - electron interaction within the Mn ions, the crystal field felt by the $\mathrm{Mn}$ ions and the hybridization of $\mathrm{Mn}_{3 d}, \mathrm{O}_{2 p}$ and $\mathrm{N}_{2 p}$ orbitals. The strong similarity between the XAS and XMCD spectra of TF - benz and TF - tpc compounds clearly suggests that the tpc functionalization does not significantly affect the electronic and magnetic properties at the Mn sites.

In Figure 3 the isotropic $\mathrm{Mn}-L_{2,3}$ absorption spectra for the TFs and sMLs of $\mathbf{1}$ and 2 are compared with a series of reference Mn - oxide compounds. The absorption spectra of the TFs $\mathbf{1}$ and $\mathbf{2}$ have almost identical lineshape suggesting that the Mn ions present the same local symmetries and comparable crystal field intensities in the two complexes. Indeed the overall site geometry extracted from crystallographic data of the $\mathrm{Mn}$ ions in $\mathbf{1}$ and $\mathbf{2}$ is the same ${ }^{24}$. The local symmetry of each Mn ion (Figure 1) is represented by a distorted octahedron with six apical atoms, five $\mathrm{O}$ atoms and one $\mathrm{N}$ atom. The main difference is that all the Mn ions are six-coordinated in 2, while four Mn ions are six coordinated and the other two are five - coordinated in $\mathbf{1}$ (one apical oxygen is missing, Figure 1). Despite the obvious inequality of $\mathrm{N}$ and $\mathrm{O}$, the very similar bond lengths and angles around the magnetic ion leads to an almost perfect equivalence of the four in - plane atoms. On the other hand, their average apical - in - plane distance ratio is 1.17 for $\mathbf{1}$ and 1.19 for $\mathbf{2}$ with a percentage difference of only $1.4 \%$. In general, such small variations of the local symmetry around the selected chemical element cannot be observed in XAS spectra. For instance, in the case of other Mn - based complexes reported by Noh et al $^{29}$, no significant variation of the spectral lineshape was found for changes below $6 \%$ in the apical - in - plane ratio.

While the XAS spectra of TFs $\mathbf{1}$ and $\mathbf{2}$ are almost identical, most interestingly the sMLs $\mathbf{1}$ and $\mathbf{2}$ (Figure 3) show sensible discrepancy at the $L_{3}$ edge with respect to the corresponding TFs, the $L_{2}$ edge being quite similar. Notably, these modifications are identical for both $\mathbf{1}$ and $\mathbf{2}$ suggesting that the same kind of perturbation affects the two differently functionalized compounds when the $\mathrm{Mn}_{6}$ molecules are deposited on gold surface. We will show in the following discussion, based on comparison of XAS spectra with those of reference manganese oxide compounds, that these modifications can be ascribed to a local structural modification of the grafted clusters, when they are deposited on the substrate.

The absorption spectra of $\mathrm{MnO}\left(\mathrm{Mn}^{\mathrm{II}}\right), \mathrm{Mn}_{2} \mathrm{O}_{3}\left(\mathrm{Mn}^{\mathrm{III}}\right)$ and $\mathrm{MnO}_{2}\left(\mathrm{Mn}^{\mathrm{IV}}\right)$ presented in Figure 3 as references were measured at the same time and in the same experimental conditions with the $\mathrm{Mn}_{6}$ derivatives. Particular attention was paid for the energy alignment of all the spectra plotted in Figure 3. The main spectral features are labelled from A to E to facilitate the comparison. For both TFs and sMLs the $\mathrm{Mn}^{\mathrm{III}}$ components $(\mathrm{B}, \mathrm{C}$ and $\mathrm{D})$ are dominant while the $\mathrm{Mn}^{\mathrm{IV}}(\mathrm{E})$ is negligible. By comparing 
the shapes of $\mathrm{MnO}\left(\mathrm{Mn}^{\mathrm{II}}\right)$ and $\mathrm{Mn}_{2} \mathrm{O}_{3}\left(\mathrm{Mn}^{\mathrm{III}}\right)$ spectra with those of the TFs, we can safely conclude that the fraction of reduced $\mathrm{Mn}^{\mathrm{II}}$ ions must be extremely small.

The superimposition of normalized XAS and XMCD spectra taken for $\mathrm{Mn}_{6}-$ tpc TFs and sMLs reported in Figure 4 allows a more accurate analysis. Four features (A, B, C and D) can be easily distinguished at the Mn $L_{3}$ edge. Two highest features B and D with comparable intensity are visible in TFs, while only D is dominant in sMLs. B and C are much higher in TFs, conversely A is higher in the sMLs. This suggests an apparent transfer of spectral weights from B and C to A in sMLs with respect to TFs which can be explained in terms of a structural modification of the Mn sites. The comparison between XMCD spectra also shows features at the same energy of A, B, C and D. The decrease of the feature $\mathrm{B}$ gives evidence for a reduction of the magnetic moment associated with the $\mathrm{Mn}^{\mathrm{III}}$ ions, as will be discussed later.

In general, the interpretation of absorption and dichroic spectra is not trivial because the lineshape changes drastically with the strength of the crystal field and spin - orbit interaction. Notwithstanding, the origin of the spectral differences between TFs and sMLs can be explained looking at the well - known $\mathrm{Mn}^{\mathrm{III}}$ based reference systems. In Figure 5 the isotropic $\mathrm{Mn}-L_{2,3}$ absorption spectra of the $\mathrm{Mn}_{6}-$ tpc TFs are compared with those of the Piedmontite ${ }^{30}$ and the $\mathrm{ZnMn}_{2} \mathrm{O}_{4}{ }^{29}$, whereas the spectra of the sMLs with those of the $\mathrm{Mn}_{2}^{\mathrm{III}} \mathrm{O}_{3}$ and $\mathrm{ZnGa}_{1} \mathrm{Mn}_{1} \mathrm{O}_{4}{ }^{29}$. The similarity between the Piedmontite, $\mathrm{ZnMn}_{2} \mathrm{O}_{4}$, and TFs absorption spectra let us suppose that the $\mathrm{Mn}$ ions posses large tetragonal distortions (i.e. Jahn - Teller distortion) as well known for polycrystalline spinel manganite oxides. This is also confirmed by the strong distortion of $\mathrm{ZnMn}_{2} \mathrm{O}_{4}$ (apical - in - plane ratio of 1.18) comparable to those of $\mathrm{Mn}_{6}$ TFs 1 and 2 (apical - in - plane ratio of 1.17 - 1.19 respectively). Conversely, the XAS spectra of the $\mathrm{ZnGa}_{1} \mathrm{Mn}_{1} \mathrm{O}_{4}$ and in particular of the $\mathrm{Mn}_{2} \mathrm{O}_{3}\left(\mathrm{Mn}^{\mathrm{III}}\right)$ with almost perfect octahedral symmetry (apical - in - plane ratio of 1.06) are more similar to the $\mathrm{Mn}_{6}-\mathrm{sMLs}$. This suggests that the absorption of the $3-\mathrm{tp}-\mathrm{CO}_{2}^{-}$functionalized $\mathrm{Mn}_{6}$ clusters on gold surface induces a structural modification of the Mn sites reducing the elongation of the $\mathrm{O}-\mathrm{Mn}-\mathrm{O}$ axes, with respect to the bulk conditions, leading to an almost octahedral symmetry. This distortion could be ascribed to the interaction with gold and / or due to the lack of the isotropic interactions with all the surrounding molecules. Notably, this mechanism occurs without affecting the oxidation state of the Mn ions because the absorption spectra of the $\mathrm{Mn}_{6}-$ sMLs and $\mathrm{Mn}_{2} \mathrm{O}_{3}$ are almost identical, as evidenced by their comparison presented in Figure 6 upper panel.

If the modifications in the XAS spectrum lead to different structural and electronic properties, those in the XMCD reflect the deviations of the magnetic properties. Comparison of the XMCD spectra (Figure 6 bottom panel) highlights that sMLs 1 and 2 are very similar to $\mathrm{Mn}_{2} \mathrm{O}_{3}$ and once again rather 
different from $\mathrm{MnO}$ also in the magnetic behaviour. Since no significant $\mathrm{Mn}^{\mathrm{II}}$ component is visible in the sMLs we can conclude that a large fraction of $\mathrm{Mn}_{6}$ molecules have been deposited on Au surface preserving the $\mathrm{Mn}^{\mathrm{III}}$ oxidation state; unlike the case for $\mathrm{Mn}_{12}$ - ac where a systematic reduction of the magnetic core occurs after the adsorption on gold ${ }^{15,16,31,32}$. The sharp feature $B$ of the reference compound $\mathrm{Mn}_{2} \mathrm{O}_{3}$ is observed in both TFs and sMLs with different intensities. Ghigna ${ }^{33}$ and Voss ${ }^{16}$ observed the peak $\mathrm{B}$ in the $\mathrm{Mn}_{12}$ - biph spectrum and they assigned it to different crystal symmetry or to a poor single crystal quality. Because in sMLs and TFs the molecules are not aligned in the same direction like in single crystals we suggest that B reflects local symmetries of the Mn sites.

The application of the XMCD spin moment sum rule for $\mathrm{Mn}^{\mathrm{III}}$ is unfeasible because the $L_{2,3}$ edges overlap due to the strong spin - orbit coupling and it is also complicated by the determination of the magnetic dipole operator $(\mathrm{Tz})^{34,35,36}$. Conversely, the orbital moment sum rule still holds and by definition it is proportional to the integral of the XMCD signal through the $L_{2,3}$ edges ${ }^{34}$. The XMCD integral curves reported in Figure 4 vanish for both TFs and sMLs of $\mathbf{1}$ and $\mathbf{2}$ giving evidence for quenched orbital moment. Hence, the total magnetic moment posses only spin contribution. Since the orbital moment is quenched for all TFs ( - benz and - tpc), we conclude that neither the functionalization nor the deposition on gold surface affects the degree of quenching of the orbital momentum at the Mn sites. Variable temperature variable field (VTVH) XMCD signal (\%) at the $L_{3}$ edge for the TFs and sMLs of $\mathbf{1}$ and $\mathbf{2}$ are plotted in Figure 7. The uncertainty is estimated to be $20 \%$ of the corresponding maximum values. The comparison between TFs and sMLs shows that: (i) as expected the dichroic signal of the TF $\mathbf{2}$ is more intense with respect to the TF $\mathbf{1}$ in agreement with the nominal values of their spin; (ii) the dichroic signal for the TFs and sMLs decreases with increasing temperature from 10 to $25 \mathrm{~K}$ due to the increased thermal fluctuation of the spin magnetic moments, (iii) upon the increase of the temperature the dichroic signal in the sMLs decreases with respect to the TFs from $30 \%$ at $10 \mathrm{~K}$ to $50 \%$ at $25 \mathrm{~K}$. Because the error bars of the TFs and sMLs isothermal curves do not overlap, the $\mathrm{Mn}^{\mathrm{III}}$ spin magnetic moment shows an effective decrease. According with the results of XAS measurements (Figure 4) this can be explained in terms of structural changes of the Mn local environment when they are grafted on gold surface in sMLs compounds. In fact such changes may lead to different relative distances and angles between Mn ions and to the modification of the Mn - Mn exchange couplings with respect to the corresponding TFs. The origin of such structural changes can be ascribed to the molecule - gold surface interactions and / or to the lack of the isotropic interactions with all the surrounding molecules. In fact, in the TFs the molecules are packed and unless the first layer, the molecules probed by XMCD in TEY mode are surrounded by others. Hence isotropic molecule - molecule interactions are expected while interactions with the surface do not play any role 
because the TFs are deposited on poor reactive HOPG surface and also because of the small sampling depth of the TEY mode (2 - 3 molecular layers) with respect to the TFs thickness. In the sMLs the molecules are surrounded by the surface on bottom, free space on top and molecules in the plane (Figure 1). Then local and molecular symmetries are driven by anisotropic molecule - molecule and molecule - surface interactions. Other effects such as preferential orientation of the molecular easy axis of the magnetisation in the plane of the surface, and high - spin to low - spin transitions driven by a competition between the inter - atomic exchange couplings and the crystal field strength must be considered. If the molecular easy axis lies preferentially in the plane of the surface, then the variable field XMCD signal is expected to be lower than the relative TFs because the magnetic field is applied perpendicular to the surface and along the molecular hard axis. This can be proven only with further studies based on grazing incident XMCD experiment although STM studies were in favour with preferential molecular axis perpendicular to the surface ${ }^{24}$. The transition of $\mathrm{Mn}^{\mathrm{III}}$ ions from high - spin to low - spin can be also disregarded because the XAS lineshape for both sMLs and TFs $\mathbf{1}$ and $\mathbf{2}$ have a well - defined multiplet structure, which is a clear fingerprint of localized 3d orbital with high - spin state. Conversely, itinerant systems with low - spin state are characterized by smooth XAS spectral lineshape ${ }^{37,38}$.

\section{Conclusions}

The electronic and magnetic properties of functionalised derivatives 1 and 2 belonging to a $\mathrm{Mn}_{6}$ MNMs family as TFs and sMLs on gold surface have been investigated by XAS and XMCD techniques. The results can be summarised as follows: i) the functionalisation does not affect the properties of the $\mathrm{Mn}_{6}$ magnetic core as confirmed by the similarity of XAS and XMCD spectra of $\mathrm{Mn}_{6}$ - benz and $\mathrm{Mn}_{6}$ - tpc as TFs; ii) a comparative analysis of XAS and XMCD spectra of $\mathbf{1}$ and 2 as TFs and as sMLs gave evidence for local distortion of $\mathrm{Mn}$ environments when $\mathrm{Mn}_{6}$ clusters were deposited on gold surface; iii) the $\mathrm{Mn}^{\mathrm{III}}$ oxidation state was preserved in sMLs $\mathbf{1}$ and $\mathbf{2}$ encouraging the synthesis of MNMs based on $\mathrm{Mn}^{\mathrm{III}}$ ions having large magnetic anisotropy; iv) the orbital moment is quenched in the TFs as well as in the sMLs; iv) VTVH XMCD curves of sMLs reveal an effective decrease of the $\mathrm{Mn}^{\mathrm{III}}$ spin magnetic moment in $\mathbf{1}$ and $\mathbf{2}$ with respect to the relative TFs. The main effect responsible for the last result might be interpreted as a consequence of ii) where the origin of the local distortions can be ascribed to the molecule - gold surface interactions and / or to the lack of the isotropic interactions with all the surrounding molecules. In order to distinguish between the these two effects, further studies based on $\mathrm{Mn}_{6}$ clusters deposited on less reactive surfaces like HOPG will be addressed. 


\section{Acknowledgements}

We are grateful to Ivan Marri for stimulating and helpful discussions. This work has been supported by FP7 - ICT FET Open "MolSpinQIP" project, contract N. 211284. ME acknowledges funding from the Spanish Ministry for Science and Innovation through grants MAT2009-13977-C03 and CSD2007-

00010. We acknowledge the European Synchrotron Radiation Facility for provision of synchrotron radiation facilities. 
Figure 1
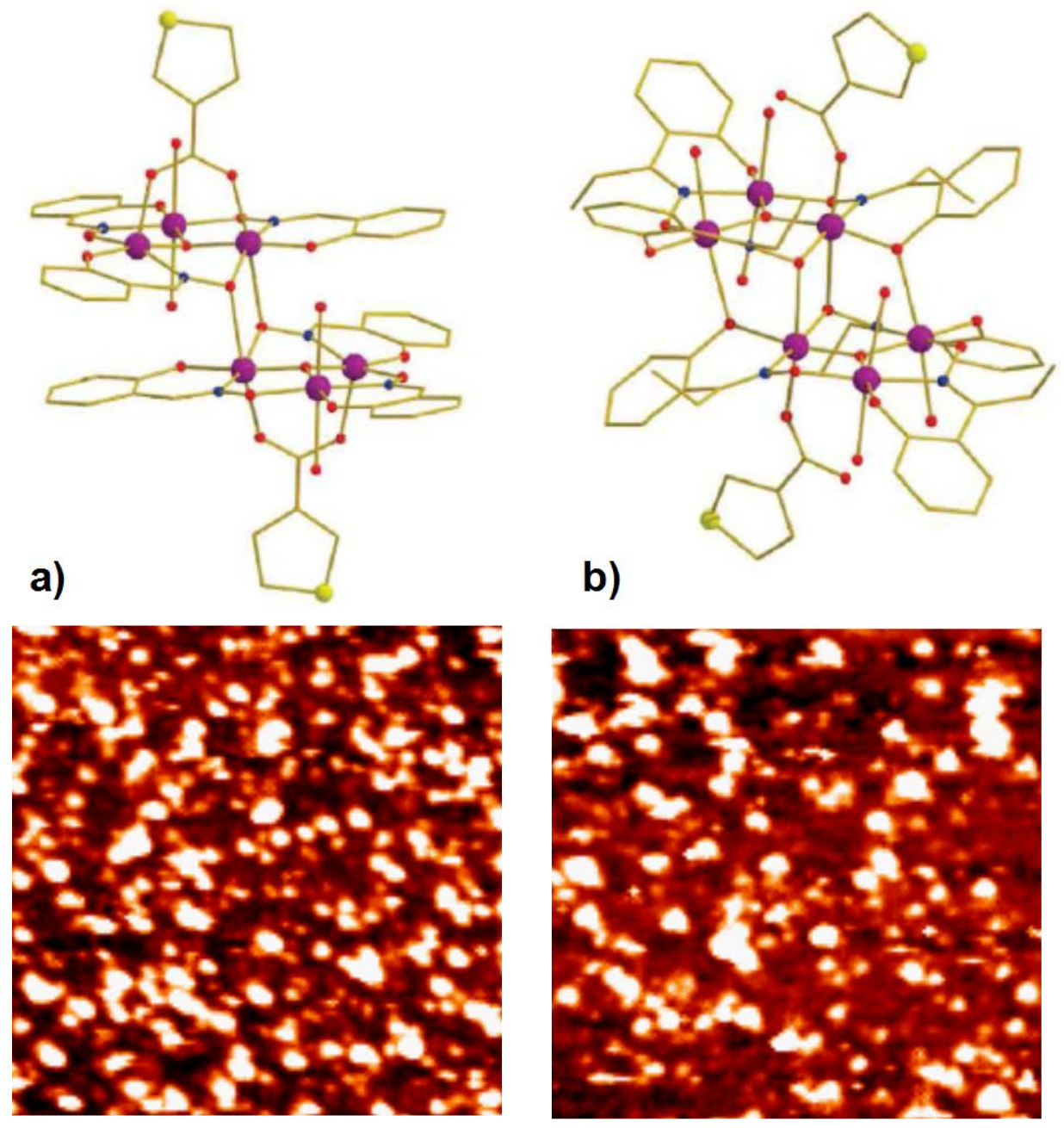

Figure 1: Upper panel: The $\mathrm{Mn}_{6}$ structures (with 3 - tp $-\mathrm{CO}_{2}{ }^{-}$functionalizations) of the derivatives 1 with $\mathrm{S}=4$ (a) and 2 with $\mathrm{S}=12$ (b). The large and medium circles depict the $\mathrm{Mn}^{\mathrm{III}}$ ions and the $\mathrm{S}$ atoms respectively. While dark and clear small circles indicate $\mathrm{N}$ and $\mathrm{O}$ atoms. The lines link the carbon atoms. $\mathrm{H}-$ atoms are omitted for clarity. Lower panel: STM images $\left(65 \times 65 \mathrm{~nm}^{2}\right)$ in constantcurrent mode of the $\mathrm{Au}(111)$ surface immersed in a solution of $\mathbf{1}$ on the left and $\mathbf{2}$ on the right. 
Figure 2
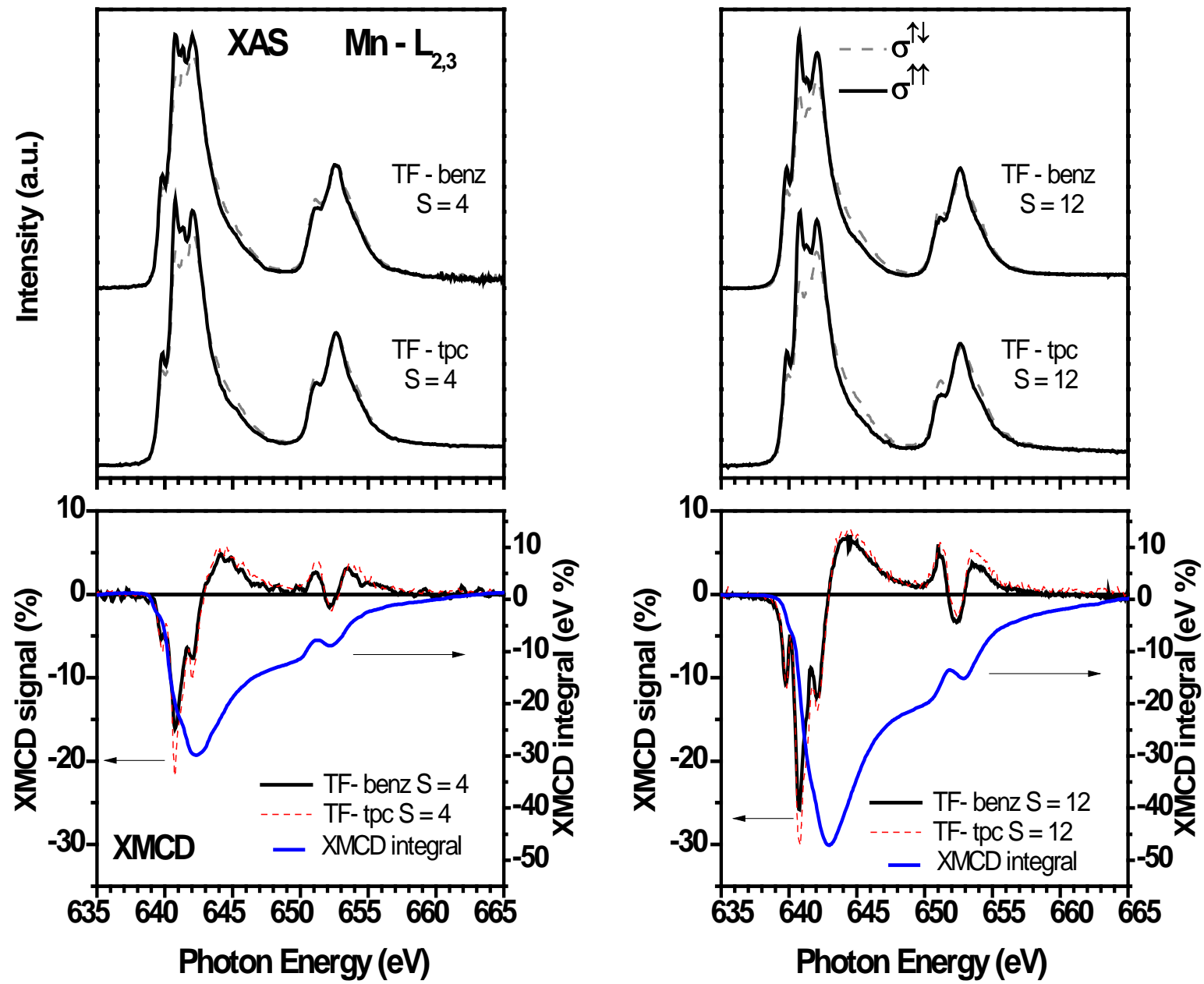

Figure 2. Upper panel: comparison between Mn $L_{2,3}$ XAS spectra, taken on TF samples with left $\left(\sigma^{\uparrow \downarrow}\right)$ and right $\left(\sigma^{\uparrow \uparrow}\right)$ circularly polarized light, of $\mathrm{Mn}_{6}-$ benz and $\mathrm{Mn}_{6}-$ tpc for both $\mathrm{S}=4$ (left) and $\mathrm{S}=12$ (right). Lower panels: dichroic spectra of $\mathrm{Mn}_{6}-$ benz and $\mathrm{Mn}_{6}-$ tpc for both $\mathrm{S}=4$ (left) and $\mathrm{S}=12$ (right). The XMCD integrals (dotted curve) relative to the tpc - functionalized $\mathrm{Mn}_{6}$ are also reported. All the spectra were taken at $10 \mathrm{~K}$ with an applied magnetic field of $5 \mathrm{~T}$. 
Figure 3

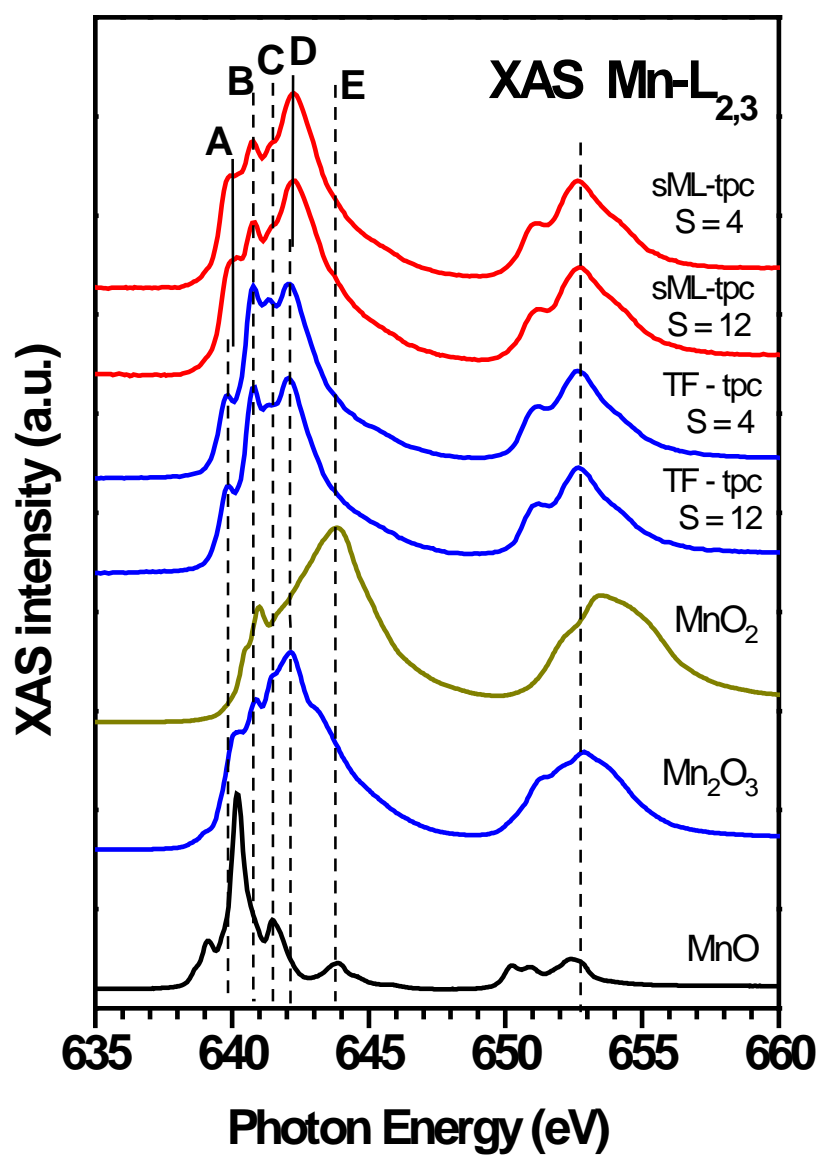

Figure 3. Comparison between the isotropic $\mathrm{Mn}-L_{2,3}$ XAS spectra of the $\mathrm{Mn}_{6} \mathrm{TF}$ and sML 1 and 2. The absorption spectra of $\mathrm{MnO}\left(\mathrm{Mn}^{\mathrm{II}}\right), \mathrm{Mn}_{2} \mathrm{O}_{3}\left(\mathrm{Mn}^{\mathrm{III}}\right)$ and $\mathrm{MnO}_{2}\left(\mathrm{Mn}^{\mathrm{IV}}\right)$ are also shown as a references. 
Figure 4
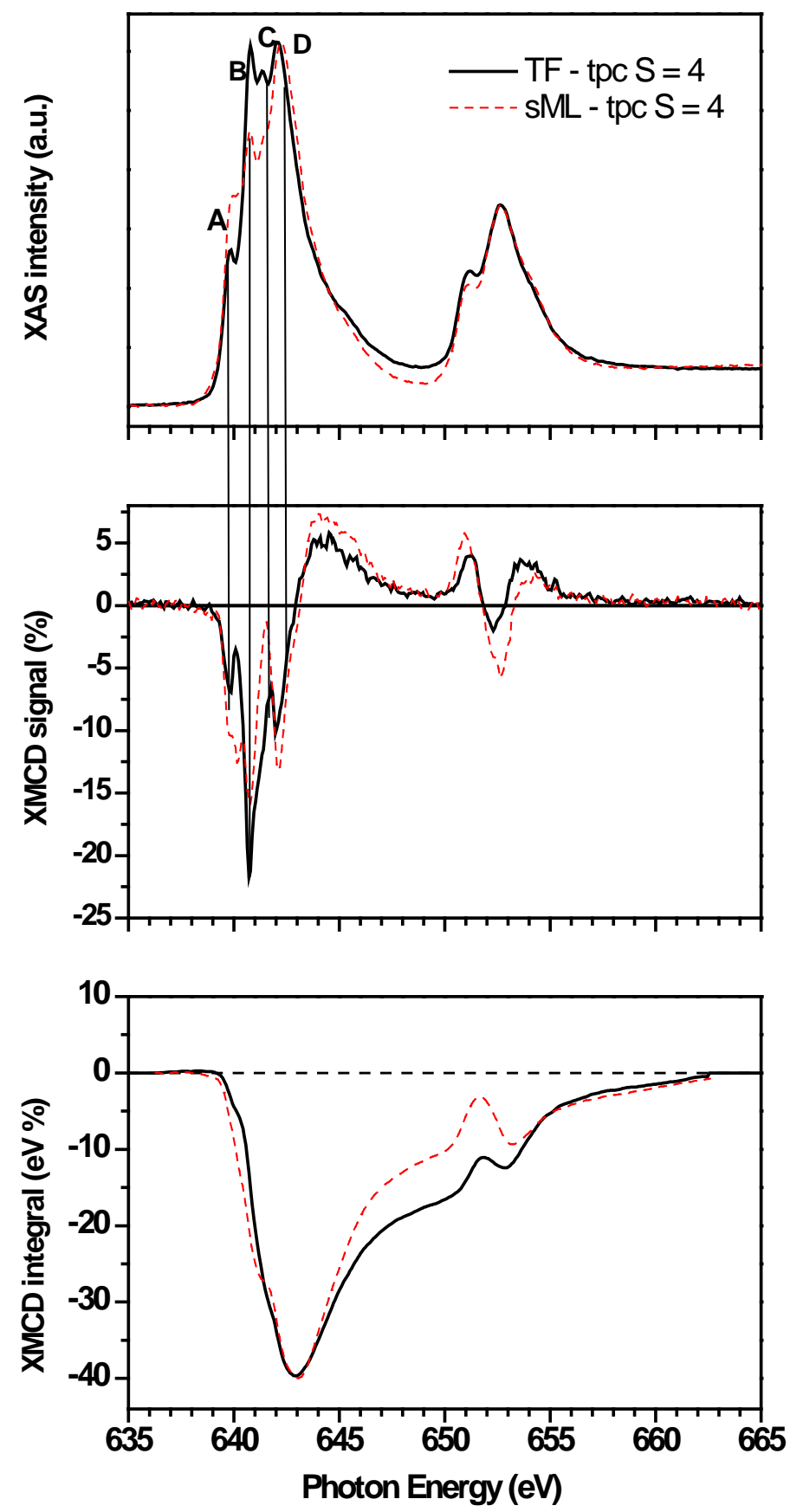

Figure 4 Direct comparison between normalized XAS and XMCD spectra of the $\mathrm{Mn}_{6}-$ tpc S $=4 \mathrm{TF}$ (black thick curve) and sML (red dashed curve). The different A, B, C and D features observed for the TF and the sML suggest deviations of the local symmetries (see the text). In the bottom panel the XMCD integrals are also shown. 
Figure 5
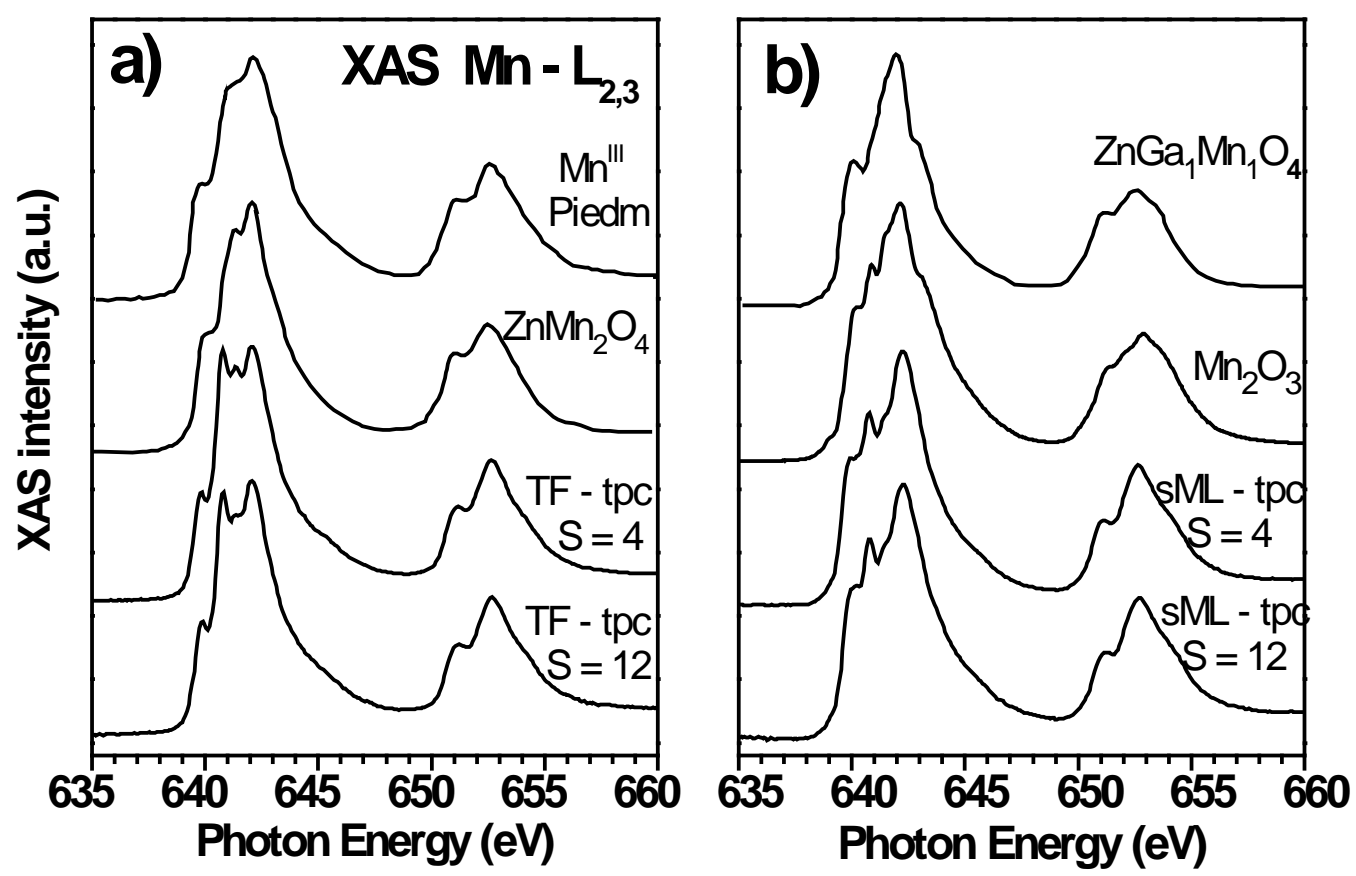

Figure 5. XAS spectra of $\mathrm{Mn}_{6}$ - tpc TF are compared with Piedmontite and $\mathrm{ZnMn}_{2} \mathrm{O}_{4}$ (a), whereas spectra of the $\mathrm{Mn}_{6}-$ tpc sML are compared with $\mathrm{Mn}_{2} \mathrm{O}_{3}\left(\mathrm{Mn}^{\mathrm{III}}\right)$ and $\mathrm{ZnGa}_{1} \mathrm{Mn}_{1} \mathrm{O}_{4}$ (b) [23, 28]. 
Figure 6

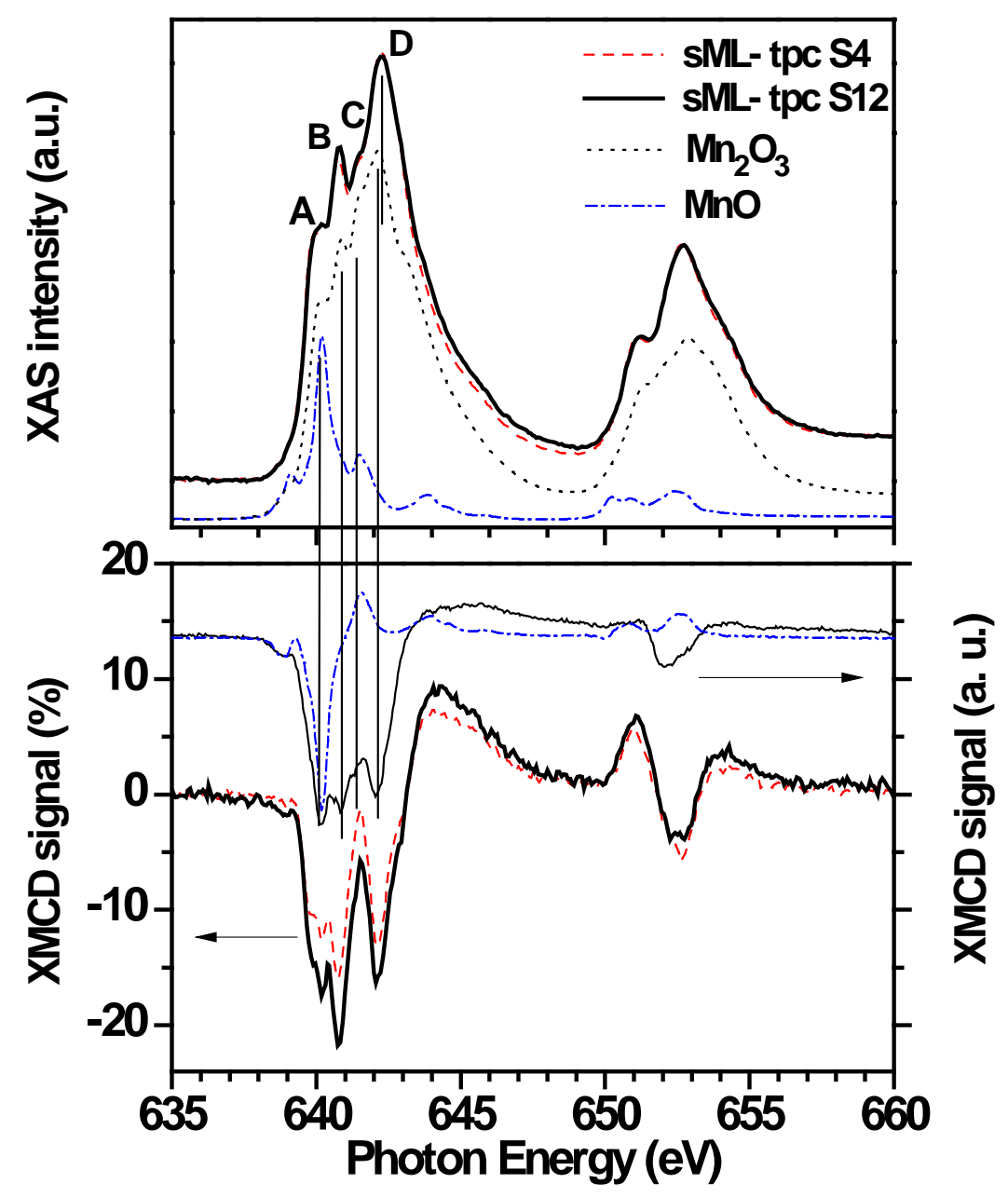

Figure 6 Normalized XAS and percentage XMCD spectra of the $\mathrm{Mn}_{6}-$ tpc sMLs, $\mathrm{S}=4$ (dash curve) and $\mathrm{S}=12$ (solid curve), are compared with the $\mathrm{Mn}_{2}{ }_{2} \mathrm{O}_{3}$ (dot curve) and the $\mathrm{Mn}{ }^{\mathrm{II}} \mathrm{O}$ (dot dash curve). The different A, B, C and D features observed in the sMLs and in the $\mathrm{Mn}_{2} \mathrm{O}_{3}$ are indicated (see the text). 
Figure 7
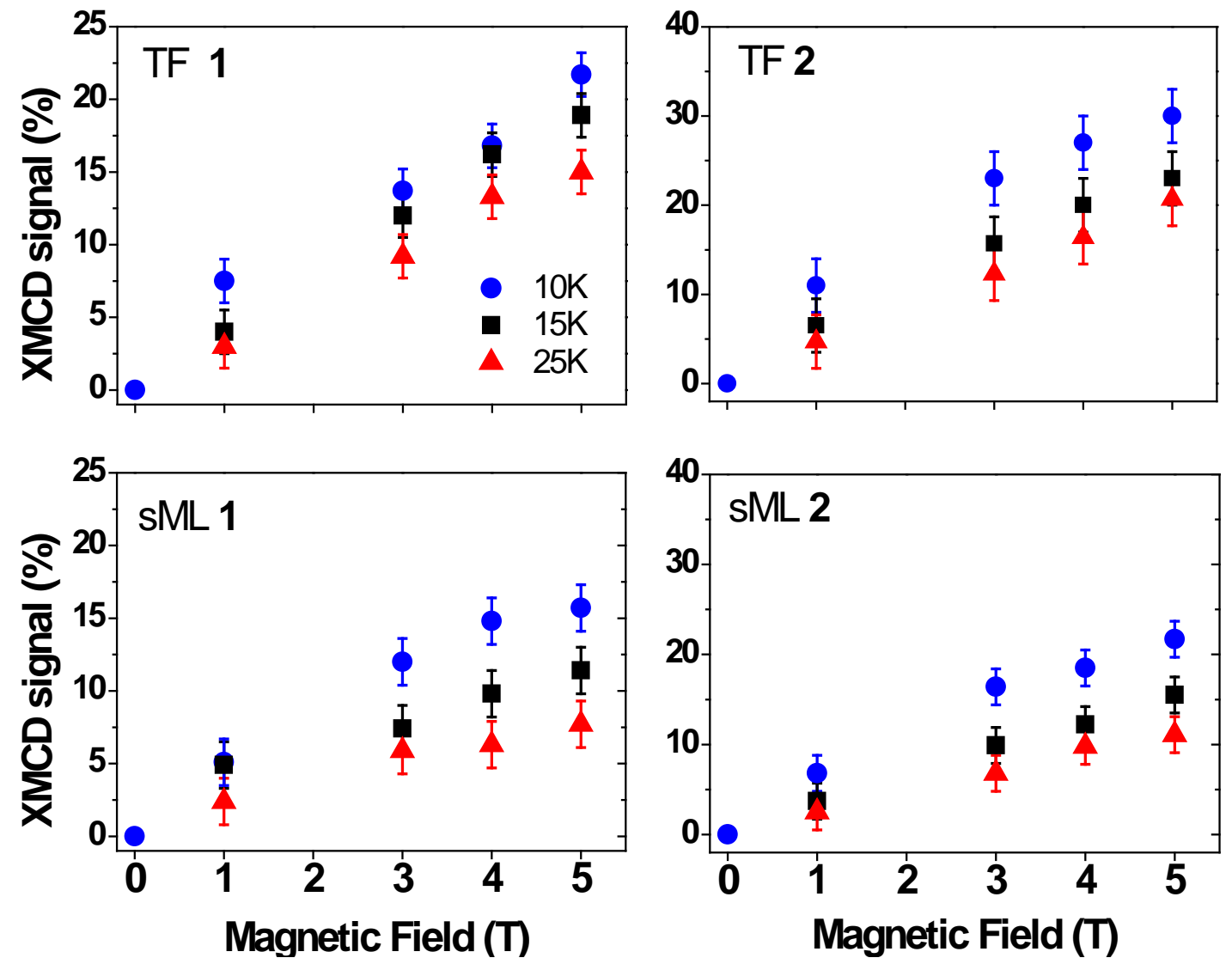

Figure 7: Variable temperature variable field XMCD signal (\%) measured for the derivatives 1 and 2 as TFs and sMLs . 


\section{References}

(1) Christou, G.; Gatteschi, D.; Hendrickson, D. N.; Sessoli, R. Mrs Bulletin 2000, 25, 66.

(2) Gatteschi, D.; Sessoli, R. Angewandte Chemie-International Edition 2003, 42, 268.

(3) Ishikawa, N.; Sugita, M.; Tanaka, N.; Ishikawa, T.; Koshihara, S. Y.; Kaizu, Y. Inorganic Chemistry 2004, 43, 5498.

(4) Ishikawa, N.; Sugita, M.; Wernsdorfer, W. Angewandte Chemie-International Edition 2005, 44, 2931.

(5) Joachim, C.; Gimzewski, J. K.; Aviram, A. Nature 2000, 408, 541.

(6) Kahn, O. VCH, Weinheim 1993.

(7) Leuenberger, M. N.; Loss, D. Nature 2001, 410, 789.

(8) Meier, F.; Levy, J.; Loss, D. Physical Review Letters 2003, 90.

(9) Sessoli, R.; Gatteschi, D.; Caneschi, A.; Novak, M. A. Nature 1993, 365, 141.

(10) Lis, T. Acta Crystallographica Section B-Structural Science 1980, 36, 2042.

(11) Friedman, J. R.; Sarachik, M. P.; Tejada, J.; Ziolo, R. Physical Review Letters 1996, 76,

3830.

(12) Thomas, L.; Lionti, F.; Ballou, R.; Gatteschi, D.; Sessoli, R.; Barbara, B. Nature 1996, $383,145$.

(13) Cavallini, M.; Gomez-Segura, J.; Ruiz-Molina, D.; Massi, M.; Albonetti, C.; Rovira, C.; Veciana, J.; Biscarini, F. Angewandte Chemie-International Edition 2005, 44, 888.

(14) Bogani, L.; Cavigli, L.; Gurioli, M.; Novak, R. L.; Mannini, M.; Caneschi, A.; Pineider, F.; Sessoli, R.; Clemente-Leon, M.; Coronado, E.; Cornia, A.; Gatteschi, D. Advanced Materials 2007, 19, 3906.

(15) Mannini, M.; Sainctavit, P.; Sessoli, R.; Moulin, C. C. D.; Pineider, F.; Arrio, M. A.; Cornia, A.; Gatteschi, D. Chemistry-a European Journal 2008, 14, 7530.

(16) Voss, S.; Fonin, M.; Rudiger, U.; Burgert, M.; Groth, U.; Dedkov, Y. S. Physical Review B 2007, 75.

(17) Inglis, R.; Taylor, S. M.; Jones, L. F.; Papaefstathiou, G. S.; Perlepes, S. P.; Datta, S.; Hill, S.; Wernsdorfer, W.; Brechin, E. K. Dalton Transactions 2009, 9157.

(18) Milios, C. J.; Inglis, R.; Vinslava, A.; Bagai, R.; Wernsdorfer, W.; Parsons, S.; Perlepes, S. P.; Christou, G.; Brechin, E. K. Journal of the American Chemical Society 2007, 129, 12505.

(19) Milios, C. J.; Vinslava, A.; Wernsdorfer, W.; Moggach, S.; Parsons, S.; Perlepes, S. P.; Christou, G.; Brechin, E. K. Journal of the American Chemical Society 2007, 129, 2754.

(20) Milios, C. J.; Vinslava, A.; Whittaker, A. G.; Parsons, S.; Wernsdorfer, W.; Christou, G.; Perlepes, S. P.; Brechin, E. K. Inorganic Chemistry 2006, 45, 5272.

(21) Milios, C. J.; Manoli, M.; Rajaraman, G.; Mishra, A.; Budd, L. E.; White, F.; Parsons, S.; Wernsdorfer, W.; Christou, G.; Brechin, E. K. Inorganic Chemistry 2006, 45, 6782.

(22) Milios, C. J.; Raptopoulou, C. P.; Terzis, A.; Vicente, R.; Escuer, A.; Perlepes, S. P. Inorganic Chemistry Communications 2003, 6, 1056.

(23) Milios, C. J.; Vinslava, A.; Wernsdorfer, W.; Prescimone, A.; Wood, P. A.; Parsons, S.; Perlepes, S. P.; Christou, G.; Brechin, E. K. Journal of the American Chemical Society 2007, 129, 6547.

(24) Moro, F.; Corradini, V.; Evangelisti, M.; Renzi, V. D.; Biagi, R.; Pennino, U. d.; Milios, C. J.; Jones, L. F.; Brechin, E. K. The Journal of Physical Chemistry B 2008, 112, 9729.

(25) del Pennino, U.; Corradini, V.; Biagi, R.; De Renzi, V.; Moro, F.; Boukhvalov, D. W.; Panaccione, G.; Hochstrasser, M.; Carbone, C.; Milios, C. J.; Brechin, E. K. Physical Review B 2008, 77.

(26) Corradini, V.; Moro, F.; Biagi, R.; del Pennino, U.; De Renzi, V.; Carretta, S.; Santini, P.; Affronte, M.; Cezar, J. C.; Timco, G.; Winpenny, R. E. P. Physical Review B 2008, 77. 
(27) Ghirri, A.; Lorusso, G.; Moro, F.; Troiani, F.; Corradini, V.; Muryn, C.; Tuna, F.; Timco, G.; Winpenny, R. E. P.; Affronte, M. Physical Review B 2009, 79.

(28) Stohr, J. Journal of Electron Spectroscopy and Related Phenomena 1995, 75, 253.

(29) Noh, H. J.; Yeo, S.; Kang, J. S.; Zhang, C. L.; Cheong, S. W.; Oh, S. J.; Johnson, P. D. Applied Physics Letters 2006, 88.

(30) Cramer, S. P.; Degroot, F. M. F.; Ma, Y.; Chen, C. T.; Sette, F.; Kipke, C. A.; Eichhorn, D. M.; Chan, M. K.; Armstrong, W. H.; Libby, E.; Christou, G.; Brooker, S.; McKee, V.; Mullins, O. C.; Fuggle, J. C. Journal of the American Chemical Society 1991, 113, 7937.

(31) Grumbach, N.; Barla, A.; Joly, L.; Donnio, B.; Rogez, G.; Terazzi, E.; Kappler, J. P.; Gallani, J. L. European Physical Journal B 2010, 73, 103.

(32) Naitabdi, A.; Bucher, J. P.; Gerbier, P.; Rabu, P.; Drillon, M. Advanced Materials 2005, $17,1612$.

(33) Ghigna, P.; Campana, A.; Lascialfari, A.; Caneschi, A.; Gatteschi, D.; Tagliaferri, A.; Borgatti, F. Physical Review B 2001, 64.

(34) Carra, P.; Thole, B. T.; Altarelli, M.; Wang, X. D. Physical Review Letters 1993, 70, 694.

(35) Crocombette, J. P.; Thole, B. T.; Jollet, F. Journal of Physics-Condensed Matter 1996, 8, 4095.

(36) Goering, E. Philosophical Magazine 2005, 85, 2895.

(37) Durr, H. A.; vanderLaan, G.; Spanke, D.; Hillebrecht, F. U.; Brookes, N. B. Physical Review B 1997, 56, 8156.

(38) Vanderlaan, G.; Thole, B. T.; Sawatzky, G. A.; Verdaguer, M. Physical Review B 1988, 37, 6587. 